Review Article

\title{
LARYNGECTOMY: FROM STRIDOR TO SURVIVAL- OUR EXPERIENCE
}

\author{
Satheesh Kumar Bhandary ${ }^{1}$, Vadish S. Bhat ${ }^{2}$, M. Shwetha Shenoy ${ }^{3}$ \\ ${ }^{1}$ Professor \& Head, ${ }^{2}$ Associate Professor, ${ }^{3}$ Sr. Resident, Department of E.N.T, \\ K.S. Hegde Medical Academy, M angalore - 575018. \\ Correspondence: \\ Satheesh Kumar Bhandary, \\ Professor \& HOD, Department of E.N.T , K.S. Hegde M edical Academy, Mangalore - 575018. \\ Mobile No. : 9845130517 E-mail : Sakubaraj@yahoo.co.
}

\begin{abstract}
:
Laryngeal cancer is among the commonest cancers in the body. It constitutes one fifth of all head and neck cancers. India accounts for $17 \%$ of the world burden. Even though it is curable in early stages, majority of them in India present in the later stages due to ignorance and poverty. The mainstay of treatment in advanced cases is total laryngectomy, with or without post operative radiotherapy which results in loss of voice. However, by speech rehabilitation and by modification of the technique in the form of near total laryngectomy, these patients can be assured of good quality disease free survival.
\end{abstract}

Keywords: Laryngeal cancer; Total Laryngectomy; Near total laryngectomy; voice rehabilitation

\section{Introduction:}

The larynx is an integral part of upper aerodigestive tract and has vital functions like protection of the lower airways, phonation and fixation of thoracic cage. Any lesion involving the larynx or any procedure that ablates the larynx, adversely affects these functions.

Larynx cancer accounts for $3.4 \%$ of all cancers and onefifth of head and neck cancers. Larynx cancer accounts for 152,000 cases globally, and India accounts for $17 \%$ of the world burden. (1) In India the population based registry estimates, 25000 new cases of laryngeal cancers and 2500 cases of hypopharyngeal cancers are added every year, that means Indian every year 50000 people are at risk of compromised voice.

The larynx is divided into sub-sites: Supraglottis, Glottis and Subglottis and malignancies involving these areas behave differently as far as the clinical presentation, spread and management are concerned(2). In the western world, glottic carcinomas constitute $55 \%$ to $75 \%$ of laryngeal malignancies. These patients present early with hoarseness as the symptom and hence the diagnosis is made in the early stages. $(3,4)$ However in India, the patients with glottis malignancy often present in the later stages because they tend to neglect the initial symptom of the disease.

Hypopharynx is closely related to larynx as the pyriform fossa, which is a part of hypopharynx shares its medial wall with the larynx. Because of the close proximity of the hypopharynx to the larynx, in the surgical management of the hypopharyngeal malignancies, larynx needs to be sacrificed as an innocent bystander.

The early laryngeal and hypopharyngeal cancers are treated by modalities like radiotherapy, partial laryngectomy procedures and endolaryngeal laser surgery whereas advanced laryngeal and hypopharyngeal tumours are best treated by laryngectomy or laryngopharyngectomy resulting in loss of natural voice.

Total laryngectomy was first performed by Theodore Billroth in 1873 (5). In this procedure the entire larynx is removed and the cut end of the trachea is brought out as a permanent stoma for respiration. Creation of a 
neopharynx allows oral feeding. However removal of larynx results in loss of natural voice which is perceived as disability in the community and also a psychosocial handicap. These patients hence require rehabilitation in the form of Oesophageal voice, Tracheo esophageal prosthesis and electrolarynx to attain alternative mode of speech.

Early laryngeal cancers are considered as curable cancers either with radiotherapy or surgery with cure rates of $80 \%$ to $95 \%$ with both forms of treatment.

Even in patients with advanced T3, T4 laryngeal cancers Total laryngectomy with post operative radiotherapy has a 5 year survival rate of $80 \%$, which overweighs the disability produced by the surgery. The treatment results of T3 and T4 tumors demonstrate better cure after surgery as compared with irradiation. Vermund found surgical cure in $59 \%$ (41 of 69 ) T3N0 and T4N0 tumors as compared with $25 \%$ (9 of 36 ) by irradiation.(5)

With the introduction of near total laryngectomy by Bruce Pearson in the 1980s, there is a ray of hope of voice conservation in advanced laryngeal and hypopharyngeal malignancis. Lesions which are suitable for near total laryngectomy are those which are well lateralized and have sufficient uninvolved mucosa for reconstruction of a neo laryngeal pathway. The survival rate after near total laryngectomy is 70 to $75 \%$ and speech acquisition is seen in $83-85 \%$ of the patients. Local recurrence of malignancy is seen in $8 \%$. (7)

In the recent years Chemoradiation is gaining larger importance even in surgically resectable advanced laryngeal and hypopharyngeal malignancies in an attempt to preserve voice. Although some tumors respond to primary chemoradiation therapy, there are many cases in which the tumor does not respond and require salvage surgical resection, which results in high surgical complications. Laryngectomy, when performed meticulously in selected indicated patients, and when these patients are rehabilitated thoroughly, can offer good quality disease free survival.

In this study, we conducted a retrospective analysis of ninety patients who underwent laryngectomy for laryngeal and hypopharyngeal cancers. These patients were studied with respect to age and sex, habits, clinical features, stage and site of the disease, type of laryngectomy done, complications of surgery and survival.

\section{Materials and methods:}

Ninety patients who underwent Laryngectomy for malignancy of the larynx and hypopharynx in the department of ENT, K.S.Hegde Hospital from the year 2000 to 2010 were identified and their records reviewed. Age and sex distribution, Clinical features, type of presentation, and history of smoking and alcohol consumption, type, site and stage of malignancy, type of laryngectomy done were recorded in a proforma. Post operatively, swallowing, speech acquisition, and complication of the procedure, disease free survival were analysed. Quality of life after surgery was recorded.

Those patients who underwent laryngectomy as a primary modality of treatment and those patients who presented with recurrent or residual lesion after chemotherapy or radiotherapy were included in the study.

\section{Results:}

In our study, of the ninety patients who underwent laryngectomy, 87 (96\%) were males and $3(4 \%)$ were females. Most of the patients were in the age group of 55-65 years. The youngest patient in our study group was aged 34 years. Among the male patients 83 (92\%) patients had history of smoking. Sixty patients (66\%) also had history of alcohol consumption. Three female patients did not have any habits. Most of the patients presented with hoarseness (58\%). Stridor, Foreign body sensation and Dysphagia were the other presenting 
complaints (Table 1). Eight patients with stridor required tracheostomy during the first visit.

The most common malignancy in the study group was Transglottic malignancy $(n=42)$ followed by supraglottis $(n=24)$ and pyriform fossa $(n=18)$, and T2 Glottis Malignancy $(n=6)$.

M ajority of the patients in our study had no lymph nodes on presentation ( $n=57)$ followed by N1 $(n=12)$ and N2a $(n=12)$, closely followed by $N 2 b \quad(n=9)$. Majority of patients with pyriform fossa malignancy in the study group had lymph node metastasis $(n=12)$. All the tumors were squamous cell carcinoma on histopathology.

Of the ninety patients, $60 \%$ underwent total laryngectomy $27 \%$ underwent near total laryngectomy and salvage surgery following radiotherapy or chemotherapy was done in $10 \%$

Average hospital stay was 12 days after surgery, in patients without complications. The hospital stay was prolonged in those patients developing complications, average stay being 22 days. Nasogastric tube feeding was started 24 hours after surgery. Oral feeding was started on $10^{\text {th }}$ postoperative day, initially with liquids, followed by semisolids and solids.

The most common complication that followed total laryngectomy was hypothyroidism $(n=45)$ (table 2). Stomal stenosis was seen in 12 patients, most of them were those who underwent Near Total Laryngectomy. None of them developed severe breathing difficulty. Eight patients developed salivary leak which was healed after conservative management in all patients after average three weeks.

Speech rehabilitation was offered to all the patients. All the patients (27\%) who underwent NTL acquired laryngeal speech. $40 \%$ percent of the patients developed oesophageal speech after speech training. Three percent required tracheoeso phageal puncture for phonation. $7 \%$ failed to acquire speech of any form, $8 \%$ were lost to follow up and $6 \%$ died of disease. Table 3.

The average three year survival in the study group was $90 \%$. And average five year survival was $80 \%$. M ajority of them continued to do their original work ( $80 \%)$, where as others who could not perform hard manual work, changed their occupation.

\section{Discussion:}

Laryngeal and hypopharyngeal malignancy accounts for one fifth of all head and neck malignancies (1). The treatment option for laryngeal malignancy revolve around various factors like the Stage of tumour, site of the tumour, lymph node metastasis, general condition of the patient and associated comorbidities (3). For advanced T3 and T4 stage laryngeal and hypopharyngeal tumours, mainstay of treatment is Laryngectomy with post operative radiotherapy.

The treatment protocols have evolved in three notable stages; the first was focused on curing patients using radical surgical procedures, mainly by total laryngectomy. The second era developed with the goal of voice preservation while using sound oncological principals. The final and current era is of organ sparing protocols utilizing a combination of radiation and chemotherapy. (4) However, chemotherapy with radiotherapy is not an option for every patient. The toxic effects, like severe nausea, fatigue, mucositis, xerostomia, nephrotoxicity and suppression of the hematopoetic and immune system may cause significant morbidity in some patients. Also, mere preservation of larynx does not ensure its function since exposure to chemotherapeutic agents and radiation results in pain, dysphagia, and continued speech disturbances due to post treatment edema and fibrosis. Even though these patients get rid of the cancer, they continue to have original symptoms as sequelae of treatment. 
Total laryngectomy is perceived as a disabling surgery, having detrimental effect on the quality of life. The disadvantages of this surgery are loss of voice, permanent stoma (fig 1), inability to perform heavy manual work and social disruption. In this study, we studied 90 patients, who underwent laryngectomy, regarding the swallowing, speech, quality of life and survival.

Among the 90 patients, 87 were males and only three were females. This male preponderance in laryngeal and hypopharyngeal cancers can be attributable to the high incidence of smoking in males.

All patients had normal swallowing after surgery.

After laryngectomy, restoration of voice remains the major challenge. After total laryngectomy, voice rehabilitation offered includes oesophageal speech, tracheoesophageal puncture (fig 4 ) and electrolarynx.

Oesophageal speech is a type of non lung powered speech, which involves swallowing of air and controlled expression of air across pharyngoesophageal segment. The vibration of this segment results in voice production.

Tracheoesophageal puncture involves shunting of air across the tracheoesophaeal fistula for phonation (8).

Electrolarynxis an electronic battery operated hand held device which produces a tone when applied in close approximation to the skin of the neck. The tone produced is then articulated and speech is produced.

Near total laryngectomy is a voice conservation surgery. It involves preservation of uninvolved vocal cord and ipsilateral functioning recurrent laryngeal nerve. Air is shunted across the myomucosal shunt (fig 3)that is created from the tracheostoma to the neopharynx, to produce lung powered by vibrating the preserved vocal cord. This surgery is oncologically sound and the quality of speech produced is good. Since it is not dependent on prosthesis it is maintenance free.

Majority of the patients who underwent total laryngectomy acquired alaryngeal speech in the form of oesophageal speech, Tracheoesophageal puncture or electrolarynx, except $8 \%$ who failed to acquire speech. All patients who underwent near total laryngectomy acquired laryngeal speech.

\section{Conclusion:}

Improving survival is the main goal in treating patients with cancer of the larynx and hypopharynx. Total laryngectomy, which is a radical procedure, has disabling outcomes like loss of speech. So, voice conservation surgeries and chemoradiation have emerged as alternative to radical surgery. However, advanced laryngeal and hypopharyngeal malignancies, still require total laryngectomy for better survival rate. When properly rehabilitated, these patients can acquire alaryngeal speech and can lead a good quality life. Various options for acquisition of speech reduce the impact of the loss.

Near total laryngectomy is an oncologically sound procedure in selected advanced cases which preserves laryngeal speech. These two modalities offer rehabilitation at no extra cost of prosthesis and recurring expenses of its maintenance

Therefore the option laryngectomy should not be deferred for patients with advanced laryngeal and hypophayngeal malignancy for the fear of loss of voice, as the disease free survival and the quality of life outperforms all the other modalities of treatment. 


\begin{tabular}{|l|l|}
\hline \multicolumn{2}{|l|}{ Table 1: Presentation of patients with laryngeal and } \\
hypopharyngeal malignancy. \\
\hline Symptoms & Number of patients \\
\hline Hoarseness of voice & 52 \\
\hline Stridor & 21 \\
\hline FBS & 27 \\
\hline Dysphagia & 27 \\
\hline Referred otalgia & 10 \\
\hline
\end{tabular}

\begin{tabular}{|l|c|}
\hline \multicolumn{2}{|c|}{ Table 2: Complicationsfollowinglaryngectomy. } \\
\hline Complications & No. of patients \\
\hline Hypocalcemia & 9 \\
\hline Pharyngocutaneous fistula & 8 \\
\hline Nodal Recurrence & 6 \\
\hline Chylous leak & 1 \\
\hline Second Primary & 3 \\
\hline Stomal Stenosis & 12 \\
\hline Hypothyroidism & 45 \\
\hline Death due to disesae & 1 \\
\hline
\end{tabular}

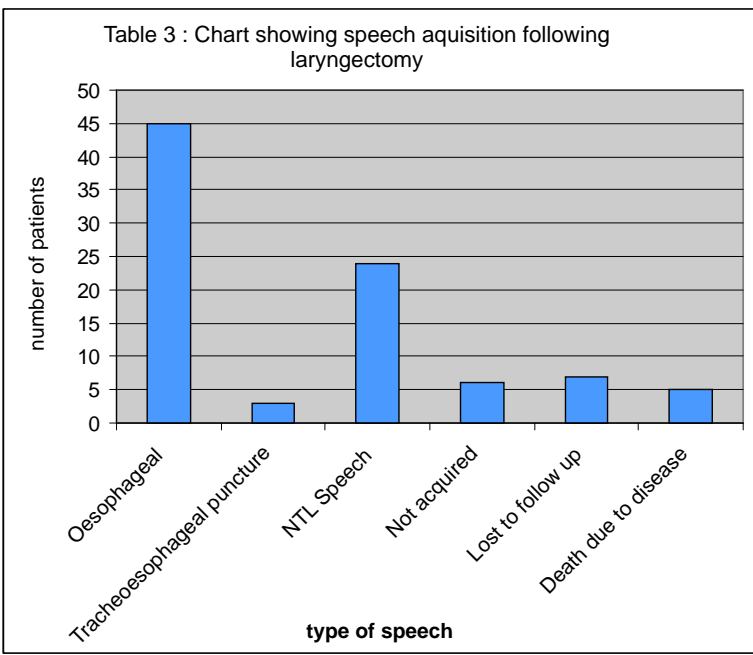

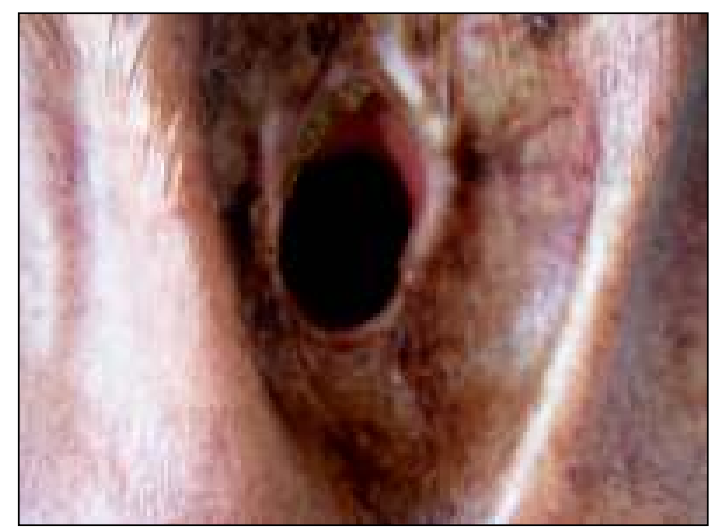

Fig 1 : Well healed stoma.

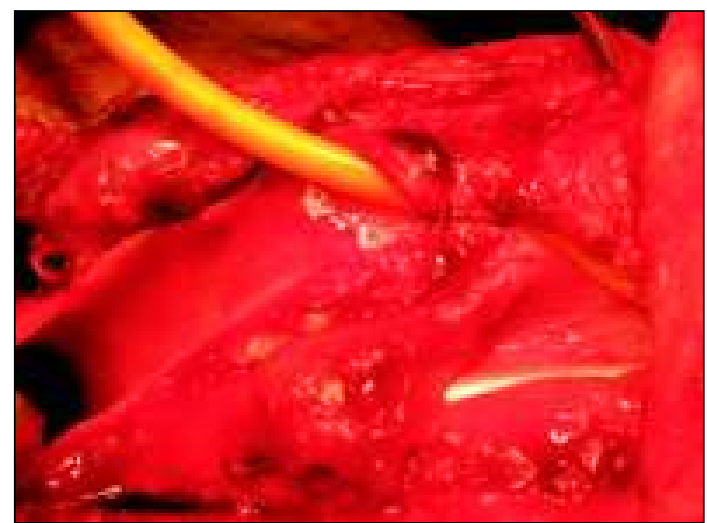

Fig 3 : Neopharyngeal stoma being constructed.

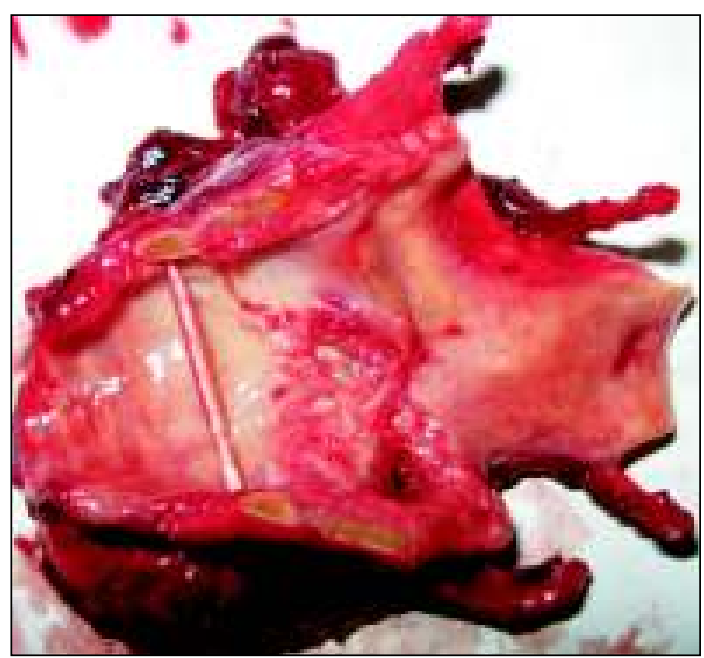

Fig 2: Laryngectomy specimen.

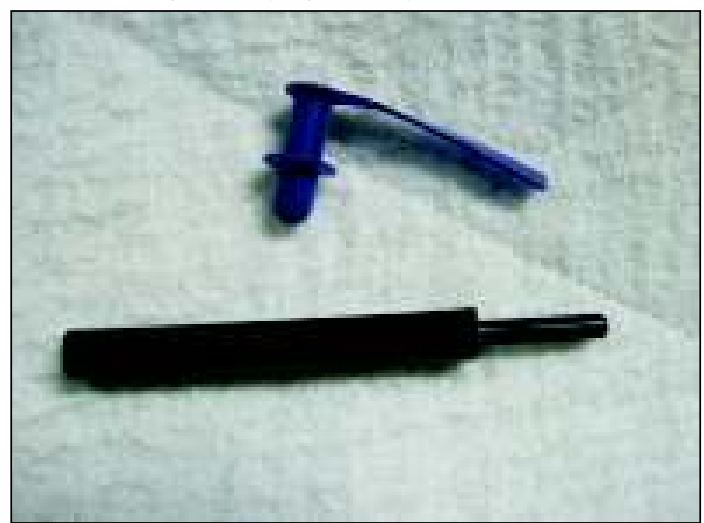

Fig 4: Tracheoesophageal prosthesis. (Nagpur prosthesis) 


\section{References:}

1. R. Sankaranarayanan, Agnihotram V. Ramanakumar, Balakrishna B. Yeole. Survival from glottic and supraglottic laryngeal carcinoma in Mumbai (Bombay), India. Oral Oncology (2003) 39 656-663.

2. Stell and Maran's head and neck surgery. 4th Ed. J. C. Watkinson, M. N. Gaze and J. A. Wilson

3. Daly C), Strong EW. Carcinoma of the glottic larynx. The American Journal of Surgery. Volume 130, Issue 4, October 1975, Pages 489-492

4. DeSanto LW, Devine KD, Lillie JC.Cancers of the larynx: glottic cancer.Surg Clin North Am. 1977 Jun;57(3):611-20.
5. Eric M. Genden, Alfio Ferlito, Carl E. Silver, Adam S. Jacobson, Jochen A. Werner, Carlos Suarez, C. Rene' Leemans, Patrick J. Bradley, Alessandra Rinaldo. Evolution of the management of laryngeal cancer. Oral Oncology (2007) 43, 431- 439

6. Vermund $\mathrm{H}$. Role of radiotherapy in cancer of the larynx in relation to TNM system of staging. Cancer M arch 1970. Vol 25. 485-504

7. Pai PS, Pradhan SA .Near-total Laryngectomy.Otorhinolaryngology Clinics(2010). Vol 2(3):247-252

8. Hall FT, O'Brien C], Clifford AR, M cNeil EB, Bron L, Jackson MA.Clinical outcome following total laryngectomy for cancer. ANZ J Surg 2003; 73:300-5 